(2) Open Access Full Text Article

ORIGINAL RESEARCH

\title{
Fentanyl Inhibits Lung Cancer Viability and Invasion via Upregulation of miR-33I-3p and Repression of HDAC5
}

This article was published in the following Dove Press journal: OncoTargets and Therapy

\author{
Shengkai Gong' \\ Liang Ying ${ }^{2}$ \\ Yu'ning Fan' \\ Zhentao Sun' \\ 'Department of Anesthesiology, Pain and \\ Perioperative Medicine, The First \\ Affiliated Hospital of Zhengzhou \\ University, Zhengzhou, Henan 450052, \\ People's Republic of China; ${ }^{2}$ Department \\ of Anesthesiology, The Second Affiliated \\ Hospital of Zhejiang University Medical \\ College, Hangzhou, Zhejiang 310009 , \\ People's Republic of China
}

Correspondence: Zhentao Sun Department of Anesthesiology, Pain and Perioperative Medicine, The First Affiliated Hospital of Zhengzhou University, No. I Jianshe Road,

Zhengzhou, Henan 450052, People's

Republic of China

Email sunzhentaozzu@|26.com
Purpose: Non-small cell lung cancer (NSCLC) accounts for more than $80 \%$ of lung cancer cases and remains the primary cause of cancer-related deaths worldwide. Fentanyl is a commonly utilized anesthetic during the process of tumor resection, and exhibits inhibitory effects on the progression of numerous cancer types, including pancreatic cancer, colorectal cancer and gastric cancer. However, the effects of fentanyl on the cell viability and invasion of NSCLC has not been investigated. Current study aimed to investigate the effects and the mechanisms underlying the effects of fentanyl on NSCLC.

Methods: The expression of $\mu$-opioid receptor (MOR) was proved by flow cytometry. The expression of microRNA-331-3p (miR-331-3p) and histone deacetylase 5 (HDAC5) in NSCLC tissues and cell lines are evaluated by reverse transcription-quantitative PCR (RT-qPCR) and Western blot, respectively. Cell viability and invasion are measured by cell counting kit-8 (CCK8) assay and transwell assay, respectively. The interaction between miR-331-3p and 3'untranslated region (UTR) of HDAC5 is predicted by TargetScan 7.1 (http://www.targetscan. org/vert_71/), validated by dual luciferase assay, RT-qPCR and Western blot.

Results: There was lower miR-331-3p expression and higher HDAC5 expression in NSCLC cell lines A549 and CALU-1 compared with BEAS-2B, which was reversed by fentanyl administration. miR-331-3p targeted 3'-UTR of HDAC5 in NSCLC cell lines A549 and CALU-1. miR-331-3p inhibitor partially abrogated the inhibitory effects of fentanyl on NSCLC cell viability and invasion by targeting HDAC5. In addition, there was higher HDAC5 expression and lower miR-331-3p expression in tumor tissues which were isolated from patients with NSCLC compared to the adjacent normal tissues, and miR-331-3p was negatively correlated with HDAC5 in NSCLC tumor tissues.

Conclusion: Fentanyl inhibits the viability and invasion of NSCLC cells by induction of miR-331-3p and reduction of HDAC5.

Keywords: non-small cell lung cancer; NSCLC, fentanyl, cell invasion, cell viability, miR331-3p, HDAC5

\section{Introduction}

Lung cancer is a highly malignant carcinoma and a cause of cancer-related mortality, with estimated mortalities of 1.1 million globally every year. ${ }^{1}$ It is well known that most cases of death from lung cancer result from metastasis. ${ }^{2}$ Based on the histological type of lung cancer, it is divided into 2 subtypes, i.e., small cell lung cancer (SCLC) and non-small cell lung cancer (NSCLC), while NSCLC accounts for approximately $80 \%$ of all patients with lung cancer. ${ }^{3}$ Based on the pathological type 
of NSCLC, it is also mainly classified into 2 subtypes, i.e., lung adenocarcinoma (LUAD) and lung squamous carcinoma (LUSC). ${ }^{4}$ With the advances achieved in therapeutic technologies including radiation-, anti-angiogenesis-, and immune-therapy, although the prognosis of patients with NSCLC has improved, their five-year survival rate remains $<20 \%$. $^{5,6}$

Fentanyl, is an opioid agonist for $\mu$-opioid receptor (MOR), which has been commonly utilized in the areas of perioperative analgesia, clinical anesthesia, and cancer pain treatment, etc. ${ }^{7}$ Besides relieving NSCLC cancer pain, ${ }^{8,9}$ fentanyl also exhibits anticancer properties by inhibiting cancer growth and invasion, for instance, fentanyl represses the negative regulation of Ets1 on BRAF-activated lncRNA (BANCR), therefore reducing the metastasis of colorectal cancer, ${ }^{10}$ fentanyl inhibits the growth of gastric cancer cells by regulating NF- $\mathrm{NB}$ in vivo and in vitro. ${ }^{11,12}$ However, the characteristics and mechanisms of fentanyl in NSCLC cell viability and invasion remain poorly elucidated.

miRNAs are a subclass of small, endogenous RNA molecules which are lack of protein-coding activity in cells. ${ }^{13}$ Several miRNAs are aberrantly expressed in patients with NSCLC, indicating the potential importance of miRNAs during the pathogenesis of NSCLC. ${ }^{14}$ Mounting links between NSCLC and miRNAs have been reported, including decreased level of miR-21 and increased level of miR-92 in NSCLC, ${ }^{15}$ and the tumor suppresser role of miR-34 in NSCLC. ${ }^{16}$ Meanwhile, increasing links between fentanyl and miRNAs have been reported, for example, knockdown of miR-145 reverses the cardioprotective role of fentanyl, ${ }^{17}$ miR-339 decreases MOR post-transcriptionally in response to fentanyl, ${ }^{18}$ fentanyl reduces the transcription of miR-190 by promoting the phosphorylation of Yin Yang $1 .{ }^{19}$ However, the link between fentanyl and miRNA in NSCLC has not been reported. In a recent miRNA array of primary cultures of rat hippocampal neurons, fentanyl increases miR-331-3p level, ${ }^{20}$ which inhibits invasion by targeting ErbB2 and VAV2 in NSCLC. ${ }^{21}$ However, the interaction between fentanyl and miR-331-3p in NSCLC left to be uncovered, which will be investigated in current study.

\section{Materials and Methods}

\section{Cell Culture}

Human NSCLC cell line (A549 and CALU-1) and human lung epithelial cell line (BEAS-2B) were purchased from American Type Culture Collection (ATCC, Manassas, VA, USA). Cells were cultured in Roswell Park Memorial Institute
(RPMI)-1640 medium (Gibco, Carlsbad, CA, USA), which were supplemented with $1 \%$ antibiotic-antimycotic mixture (Gibco) and 10\% fetal bovine serum (FBS; Hyclone, Logan, UT, USA). The cultures were maintained in the atmosphere with humidified $5 \% \mathrm{CO}_{2}$ and $95 \%$ air at $37^{\circ} \mathrm{C}$.

\section{Cell Transfection and Treatment}

miR-331-3p inhibitor (5'-UUCUAGGAUAGGCCCAGGG GC-3'), negative control (NC) inhibitor (5'-CAGUACUUUU GUGUAGUACAA-3'), miR-331-3p mimic (sense: 5'GCCCCUGGGCCUAUCCUAGAA-3'; antisense: 5'CUAGGAUAGGCCCAGGGGCUU-3'), NC mimic (sense: 5'-UUCUCCGAACGUGUCACGUTT-3'; antisense: 5'ACGUGACACGUUCGGAGAATT-3'), siRNA-NC (sense: 5'-CGUAAUUGUACUGGUCCGAAUUGC-3'; anti-sense: 5'-GCAAUUCGGACCAGUACAAUUACG-3'), siRNAHDAC5-1 (sense: 5'-CAUUGCCCACGAGUUCUCACCU GAU-3'; anti-sense: 5'-AUCAGGUGAGAACUCGUGGG CAAUG-3') and siRNA-HDAC5-2 (sense: 5'-CAGCAUG ACCACCUGACAATT-3'; antisense: 5'-UUGUCAGGUGG UCAUGCUGTT-3') were purchased from GenePharma (Shanghai, China). For transient transfection, A549 $\left(2 \times 10^{5}\right.$ cells/well) and CALU- 1 cells $\left(2 \times 10^{5}\right.$ cells/well $)$ were seeded into the 6-well plate, then transfected with miR-331-3p mimic (40 $\mathrm{nM})$, miR-331-3p inhibitor (80 $\mathrm{nM})$, and/or siRNAHDAC5 $(30 \mathrm{nM})$ and their NCs by Lipofectamine 2000 (Invitrogen, Carlsbad, CA, USA) following the manufacturer's instructions.

Following the transfection, cells were treated fentanyl (Sigma, St. Louis, MO, USA) at different final concentrations of $0.8,2,5,12.5$, and $31 \mathrm{ng} / \mathrm{mL}$ for different time points of $12 \mathrm{~h}, 24 \mathrm{~h}$ or $48 \mathrm{~h}$, which was in the same range as the pharmacologic blood levels $(4.3 \pm 2.2 \mathrm{ng} / \mathrm{mL})$ achieved with intravenous injection of fentanyl $(5 \mu \mathrm{g} / \mathrm{kg})$ in human beings as a previous report. ${ }^{22}$

\section{Flow Cytometry}

Flow cytometry analysis of A549 and CALU-1 cells labeling MOR with purified ab10275 (Abcam) at 1/150 dilution (Blue). A549 and CALU-1 cells were fixed by $4 \%$ paraformaldehyde (PFA) and permeabilized by $90 \%$ methanol. A Goat anti rabbit IgG H\&L (FITC) (ab6717, Abcam) secondary antibody was used at $1 / 2000$. Isotype controlRabbit monoclonal IgG (Red).

\section{qRT-PCR}

Total RNA was isolated from cells and tissues by TRIzol (Invitrogen) following the manufacturer's instructions. The 
complementary DNA (cDNA) was synthesized by reversetranscription with PrimeScript RT Reagent Kit (TaKaRa, Dalian, China) following the manufacturer's instructions, and qRT-PCR was carried out by SYBR Premix ExTaq (TaKaRa) on the Stratagene Mx3000P Real-Time PCR System (Agilent Technologies, Santa Clara, CA, USA) in accordance with the manufacturer's instructions with the following thermocycling conditions: $95^{\circ} \mathrm{C}$ for $5 \mathrm{~min}$, followed with 36 cycles of $95^{\circ} \mathrm{C}$ for $10 \mathrm{sec}, 60^{\circ} \mathrm{C}$ for $30 \mathrm{sec}$, and $72^{\circ} \mathrm{C} 30 \mathrm{sec}$. The primer sequences were as followed: HDAC5 forward, 5'-GTGACACCGTGTGGAATGAG-3' and reverse 5'-AGTCCACGATGAGGACCTTG-3'; GAPDH forward, 5'-GAAGGTGAAGGTCGGAGTC-3' and reverse: 5'-GAAGATGGTGATGGGATTTC-3'; U6 forward, 5'-GCTTCGAGGCAGGTTACATG-3' and reverse, 5'-GCAACACACAACATCTCCCA-3'; miR-331-3p forward, 5'-GAGCTGAAAGCACTCCCAA-3' and reverse 5'CACACTCTTGATGTTCCAGGA-3'. Expression level of miR-331-3p was normalized against U6. Expression level of HDAC5 was normalized against GAPDH. The relative gene expressions were calculated by the method of $2^{-\Delta \Delta C T .23}$

\section{Western Blot}

Total protein was extracted from cells and tissues by radioimmunoprecipitation assay (RIPA) lysis buffer (Beyotime Biotechnology, Shanghai, China) which was supplemented with protease inhibitors (Roche, Basle, Switzerland) following the manufacturer's instructions. Each protein sample was quantified by $\mathrm{BCA}^{\mathrm{TM}}$ (Pierce, Appleton, WI, USA) according to the manufacturer's instructions. Protein samples $(15 \mu \mathrm{g})$ were separated by $8 \%$ SDS-PAGE, and transferred to polyvinylidene difluoride (PVDF) membrane. Primary antibodies against HDAC5 (Cat no. ab1439) and GAPDH (Cat no. ab9485) were purchased from Abcam (Cambridge, MA, USA) and dissolved in 5\% blocking buffer at a dilution of 1:1,000. The membranes were incubated with the above antibodies at $4{ }^{\circ} \mathrm{C}$ overnight, following with $1 \mathrm{~h}$ of incubation at room temperature with goat anti-rabbit horseradish peroxidase (HRP)-tagged secondary antibody (Cat no. ab205718, dilution: 1: 5000, Abcam). Next, the PVDF membranes were added with Immobilon Western Chemiluminescent HRP Substrate (200 $\mu \mathrm{L}$, Millipore). At last, each signal was captured, and the intensity of each band was calculated by Image Lab $^{\text {TM }}$ (Bio-Rad, Shanghai, China).

\section{Cell Viability Assay}

Cell viability of A549 and CALU-1 cell lines was measured by a cell counting kit-8 (CCK-8; Dojindo Molecular Technologies, Gaithersburg, MD, USA) following the manufacturer's instructions. Briefly, A549 cells $\left(5 \times 10^{3} /\right.$ well) and CALU- 1 cells $\left(5 \times 10^{3} /\right.$ well) were seeded into the 96-well plates, and were transfected with miR-331-3p inhibitor, and/or siRNA-HDAC5, and/or exposed to fentanyl. At 24,48 or $72 \mathrm{~h}$ later, CCK-8 solution $(10 \mu \mathrm{L})$ was added into the cells, following incubation for $1 \mathrm{~h}$ in humidified $95 \%$ air and $5 \% \mathrm{CO}_{2}$ at $37^{\circ} \mathrm{C}$. At last, the absorbance at $450 \mathrm{~nm}$ was measured with a microplate reader (Bio-Rad, Hercules, CA, USA). The 50\% inhibitory concentration (IC50) of fentanyl in A549 and CALU-1 cell lines was accordingly detected.

\section{Cell Invasion Assay}

The Transwell chamber (EMD Millipore, Billerica, MA, USA) with an $8-\mu \mathrm{m}$ pore polycarbonate membrane filter, was applied for the invasion assay in A549 and CALU-1 cell lines. The filter was inserted to the 24-well chamber which was pre-coated with Matrigel $(60 \mu \mathrm{L} /$ well, BD Biosciences, San Jose, CA, USA). Next, $5 \times 10^{5}$ A549 and $5 \times 10^{5}$ CALU- 1 cells which were suspended in $200 \mu \mathrm{L}$ serum-free RPMI1640 medium, were mixed into the upper chamber, while $600 \mu \mathrm{L}$ RPMI1640 DMEM and 10\% FBS were mixed into the lower chamber. Then, the chamber was incubated in humidified $95 \%$ air and $5 \% \mathrm{CO}_{2}$ for $24 \mathrm{~h}$ at $37^{\circ} \mathrm{C}$. Thereafter, cells on the upper membrane were scraped softly. And the cells traversed the membrane were fixed by $4 \%$ methanol (NIST, USA) for $30 \mathrm{~min}$, followed with staining by $0.1 \%$ crystal violet (Merck, Darmstadt, Germany) for 30 min. At last, the images were captured by an inverted microscope (Olympus, Tokyo, Japan).

\section{Dual-Luciferase Reporter Activity Assay}

The link between HDAC5 3'-UTR and miR-331-3p was predicted by TargetScan (Version 7.1, http://www.targets can.org/vert 71/). The sequence of the HDAC5 3'-UTR containing the predicted miR-331-3p binding site was cloned into pGL2-Basic Vector (Promega, Madison, WI, USA), and named as HDAC5-WT, while mutated HDAC5 3'-UTR which was obtained by introducing two site mutations into pGL2-HDAC5 plasmid with a Quick Site-directed mutation kit (Agilent Technologies, Inc.) was used as NC (HDAC5-MUT). Next, the vector (pGL2-HDAC5-WT or pGL2-HDAC5-MUT) was co-transfected with miR-331-3p 
mimic and miR-NC mimic into A549 and CALU-1 cells. The A549 and CALU-1 cells were harvested at $48 \mathrm{~h}$ after transfection, next, the luciferase activity was measured by the Dual Luciferase Reporter Assay System (Promega) following the manufacturer's instructions. Firefly luciferase was normalized to Renilla luciferase (Promega).

\section{Tissue Samples}

A total of 37 cases of tumor tissues and 37 cases of the adjacent normal tissues were extracted from 37 patients who were diagnosed with NSCLC and underwent surgery in The First Affiliated Hospital of Zhengzhou University between Oct, 2015 and Dec, 2017. No patient had received any radiotherapy or chemotherapy before the tumor resection. All patients agreed to the use of tissues for scientific research. And all patients provided informed consent, in accordance with the Declaration of Helsinki. Current study was approved by the Ethics Committee of The First Affiliated Hospital of Zhengzhou University.

\section{Statistical Analyses}

Results were expressed as mean \pm standard deviation (SD). Statistical analyses were carried out by GraphPad 6.0 software (GraphPad Software, San Diego, CA, USA). Differences between 2 groups were analyzed by Student's t-test, differences among 3 or more groups were analyzed by one-way

A

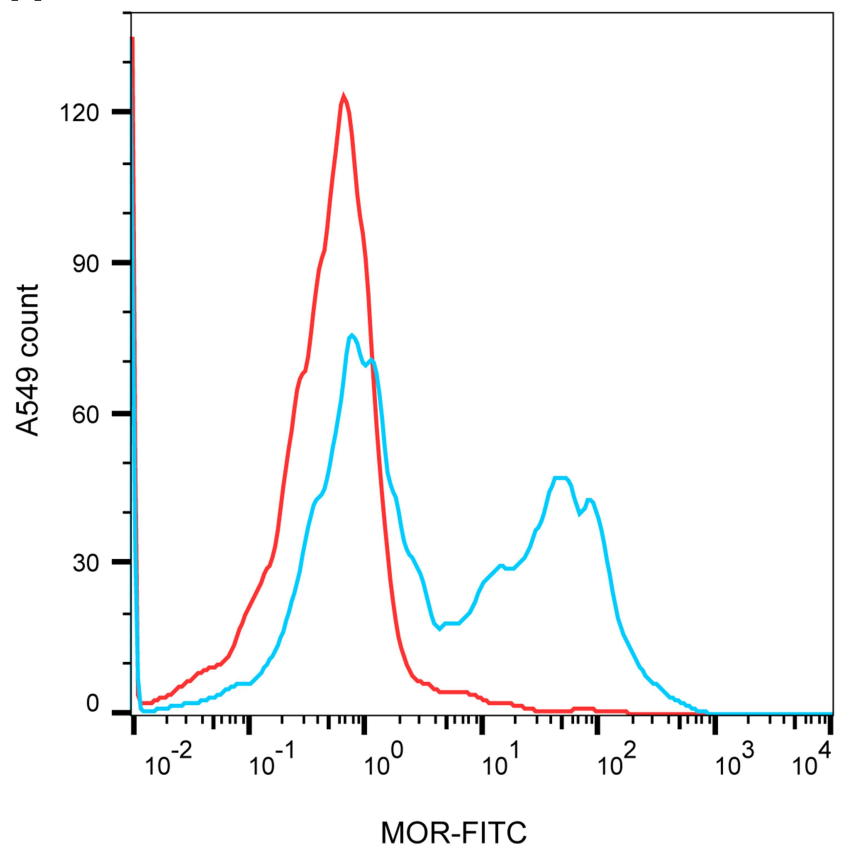

analysis of variance and Tukey's test. Pearson correlation test was used to analyze the link between miR-331-3p and HDAC5 mRNA level in the tumor tissues from patients with NSCLC. Chi-square test was used to analyze the association between miR-331-3p and clinicopathological characteristics. Values of $\mathrm{p}<0.05$ were statistically significant.

\section{Results}

\section{Fentanyl Inhibits the Viability and Invasion of A549 and CALU-I Cells}

Firstly, flow cytometry analysis proved the expression of MOR in A549 and CALU-1 cells (Figure 1A and B). Thereafter, the effects of fentanyl in A549 and CALU-1 cells were detected.

CCK-8 assay revealed that the IC50 of fentanyl treatment for $48 \mathrm{~h}$ was $5.4053 \mathrm{ng} / \mathrm{mL}$ and $4.5161 \mathrm{ng} / \mathrm{mL}$ for A549 and CALU-1, respectively (Figure 2A); additionally, fentanyl (5 $\mathrm{ng} / \mathrm{mL}$ ) inhibited cell viability of A549 and CALU-1 cells from $24 \mathrm{~h}$ to $72 \mathrm{~h}$ (Figure $2 \mathrm{~B}$ and $\mathrm{C}$ ), indicating the inhibitory function of fentanyl on A549 and CALU-1 cells in a dose- and time- dependent manner. Consequently, fentanyl ( $5 \mathrm{ng} / \mathrm{mL}, 48 \mathrm{~h}$ ) was used for the subsequent experiments.

Meanwhile, transwell assay exerted that A549 and CALU-1 cells in fentanyl group showed a decreased number of invasive cells relative to control group (Figure 2D and E).

B

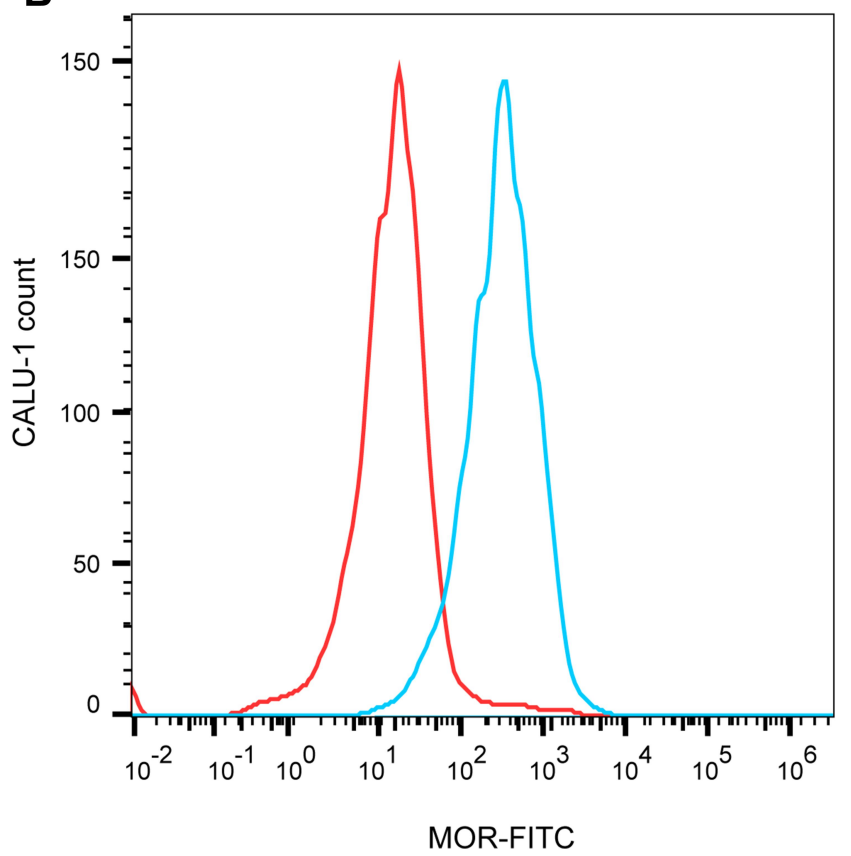

Figure I MOR is expressed in NSCLC cells. The expression of MOR in A549 and CALU-I cells was detected by flow cytometry (A, B). 

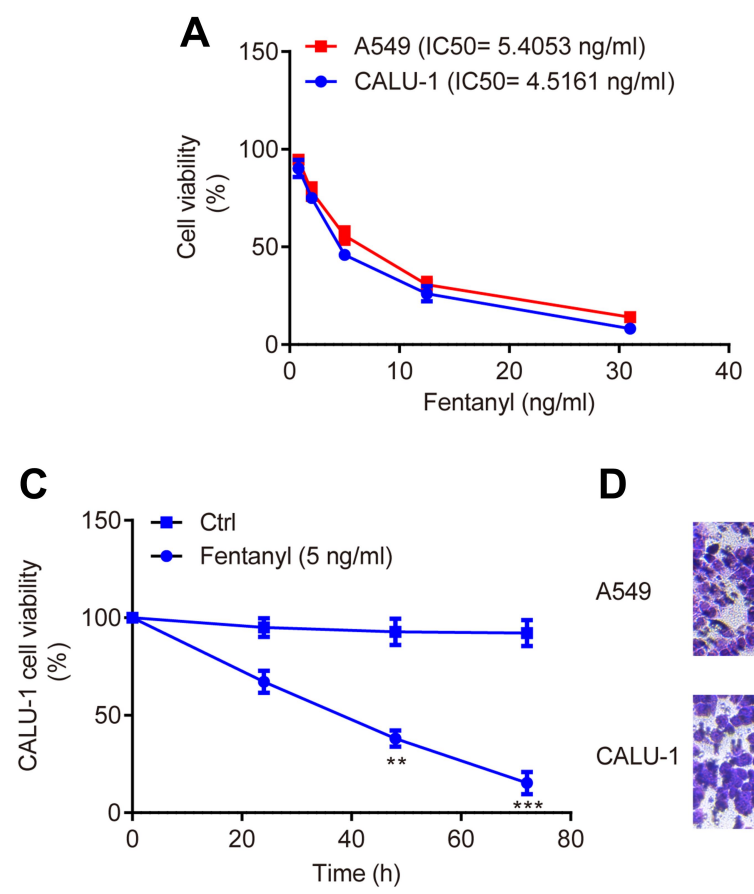

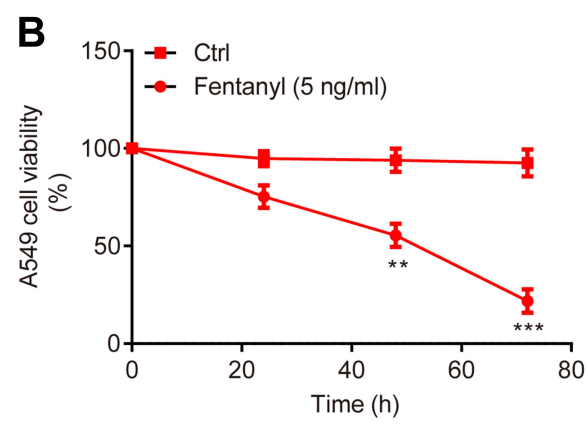

D Ctrl
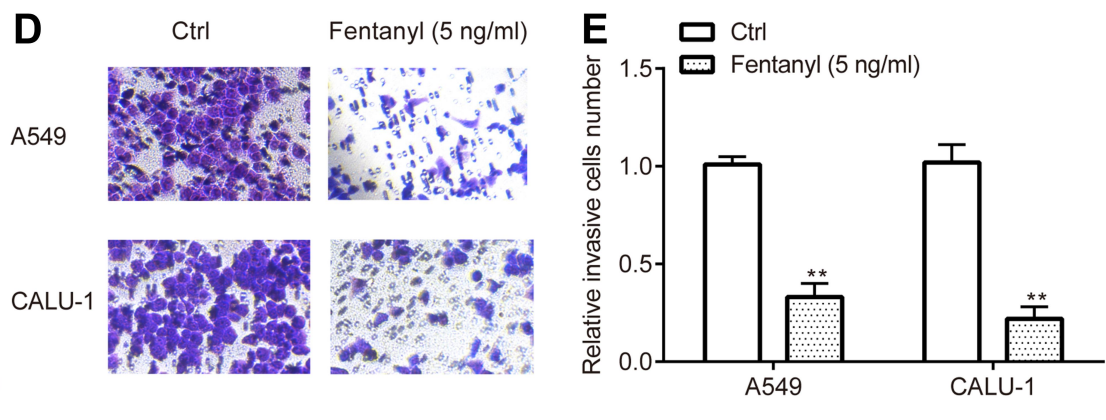

Figure 2 Fentanyl inhibits NSCLC cell viability and invasion. The effects of different concentrations and time points of fentanyl treatment on A549 and CALU-I cells were detected by CCK-8 assay (A, C). A549 and CALU-I cell invasion was determined by Transwell assay (D, E). **p $<0.01$, ***p $<0.001$, fentanyl compared with ctrl.

\section{Fentanyl Promotes miR-33I-3p}

\section{Expression in A549 and CALU-I Cells}

Next, RT-qPCR revealed that A549 and CALU-1 cells exerted lower miR-331-3p level relative to BEAS-2B (Figure 3A), while A549 and CALU-1 cells in fentanyl group showed higher miR-331-3p level relative to control group (Figure 3B).

Prior to investigating the precise function of miR-331$3 p$, the successful transfection of miR-331-3p mimic and inhibitor into A549 and CALU-1 cells was verified. RTqPCR exhibited that A549 and CALU-1 cells in miR-331$3 p$ mimic group revealed higher miR-331-3p level relative to miR-NC mimic group (Figure 3C), while those in miR331-3p inhibitor group showed lower miR-331-3p level relative to miR-NC inhibitor group (Figure 3D).

\section{miR-33 I-3p Targets HDAC5 in A549 and CALU-I Cells}

The current study aimed to determine the oncogenes that could be targeted by miR-331-3p. By using the online software TargetScan 7.1 (http://www.targetscan.org/vert 71/)), 3'UTR of HDAC5 was found to include the complementary sites for miR-331-3p (Figure 4A).

Furthermore, dual-luciferase reported assay revealed that miR-331-3p mimic inhibited the relative luciferase activity in
A549 and CALU-1 cells transfected with pGL2-HDAC5-WT but failed in those transfected with pGL2-HDAC5-MUT (Figure 4B). In addition, RT-qPCR and Western blotting demonstrated that, A549 and CALU-1 cells transfected with miR-331-3p mimic exhibited lower mRNA and protein levels of HDAC5 relative to miR-NC mimic group (Figure 4C-E).

\section{Fentanyl Decreases HDAC5 Expression in A549 and CALU-I Cells}

Next, RT-qPCR and Western blotting revealed that A549 and CALU-1 cells exhibited higher HDAC5 mRNA and protein level relative to BEAS-2B (Figure $5 \mathrm{~A}-\mathrm{C}$ ).

Then, RT-qPCR and Western blotting exerted that A549 and CALU-1 cells in fentanyl group showed lower HDAC5 mRNA and protein level relative to control group (Figure 5D-F).

\section{Fentanyl Inhibits A549 and CALU-I Cell Viability and Invasion by Regulation of miR-33I-3p and HDAC5}

Prior to measuring the effects of HDAC5, the successful transfection of siRNA-HDAC5-1 and siRNA-HDAC5-2 into A549 and CALU-1 cells was verified. RT-qPCR and Western blotting exerted that A549 and CALU-1 cells in siRNA-HDAC5-1 and siRNA-HDAC5-2 group showed 

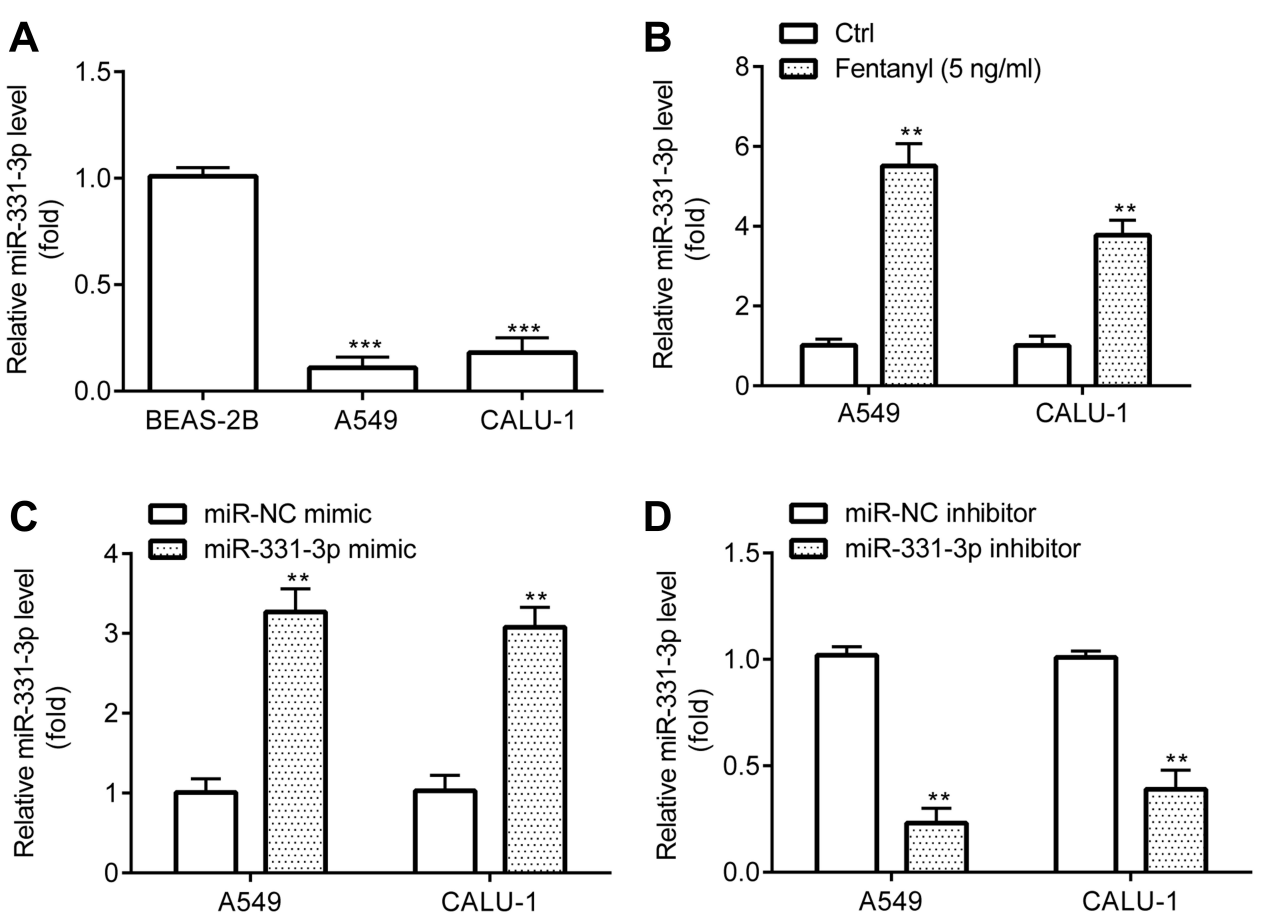

Figure 3 Fentanyl increases miR-33I-3p expression in NSCLC cells. miR-33I-3p expression was detected by RT-qPCR (A-D). **p < 0.0I, Fentanyl compared with ctrl. ***p $<0.00$ I, A549 or CALU-I cells compared with BEAS-2B.
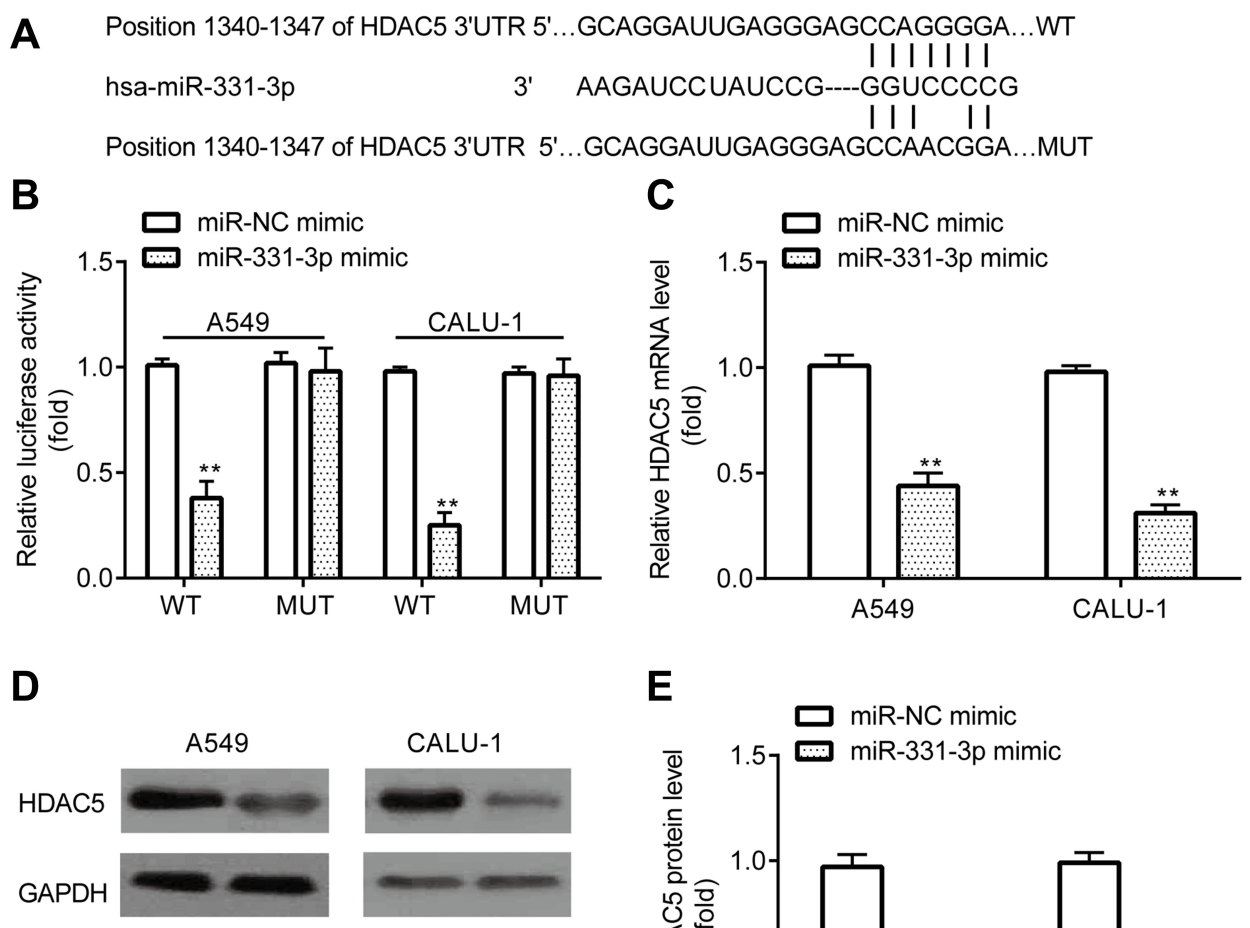

miR-NC miR-331-3p miR-NC miR-331-3p

E

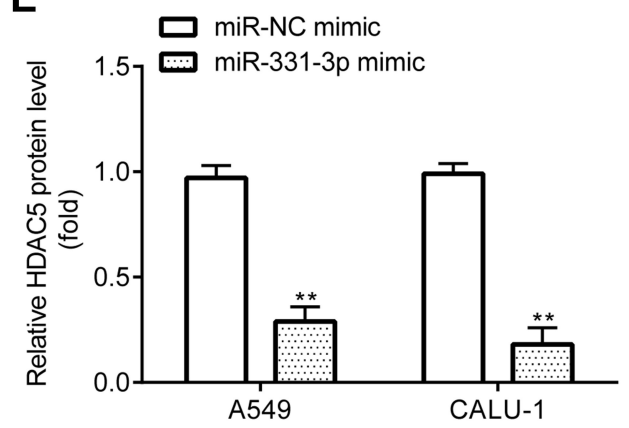

Figure 4 miR-33I-3p targets HDAC5 in NSCLC cells. TargetScan showed that the 3'UTR of HDAC5 included the complementary sites for miR-33I-3P (A). Dual-luciferase reporter assay demonstrated detected the interaction between miR-33I-3p and HDAC5 (B). RT-qPCR and Western blot were used to detect mRNA and protein levels of HDAC5 in A549 and CALU-I cells $(\mathbf{C}-\mathbf{E})$. ${ }^{* *} \mathrm{p}<0.01$, miR-33I-3p mimic compared with miR-NC mimic. 

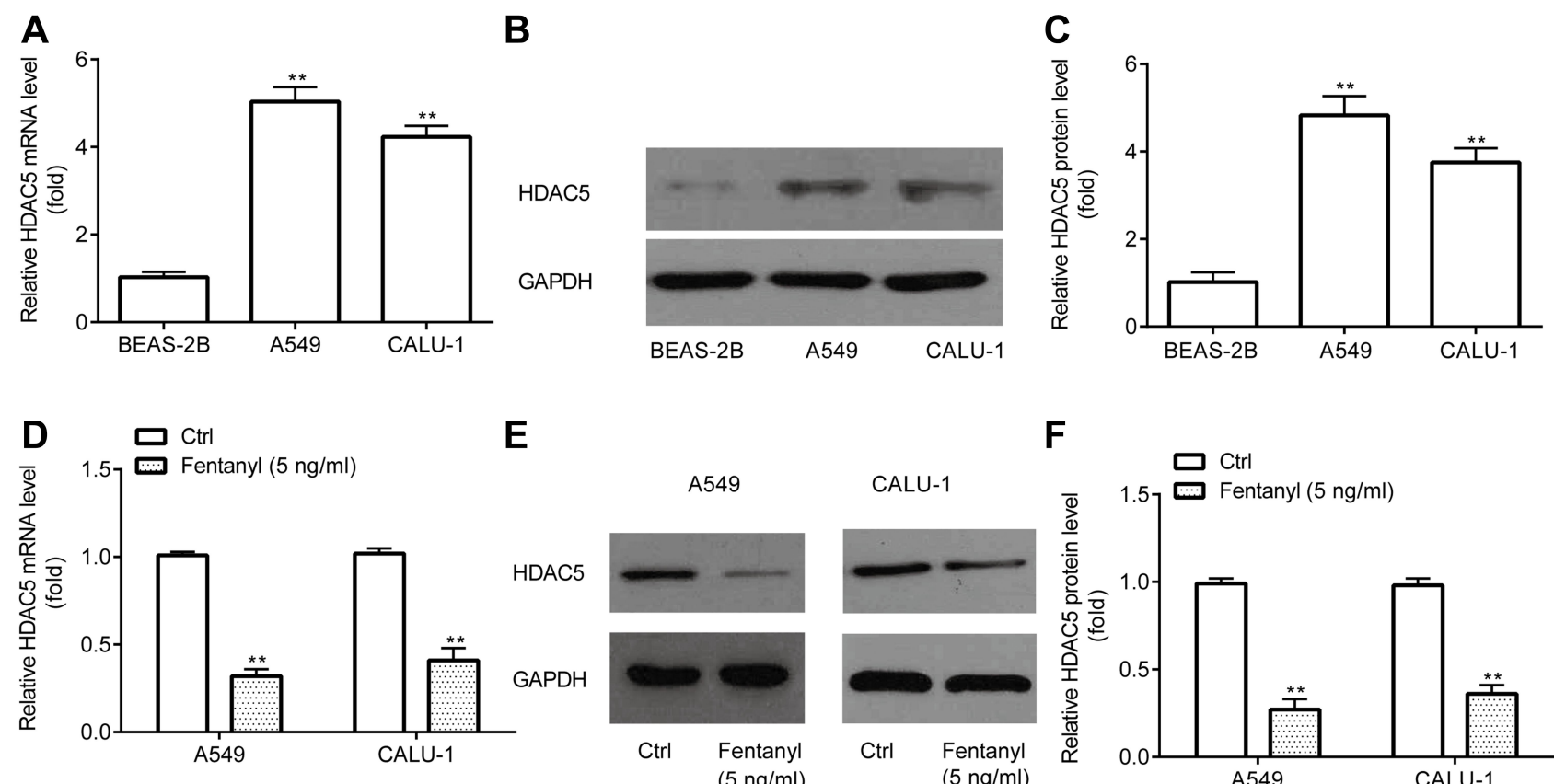

E

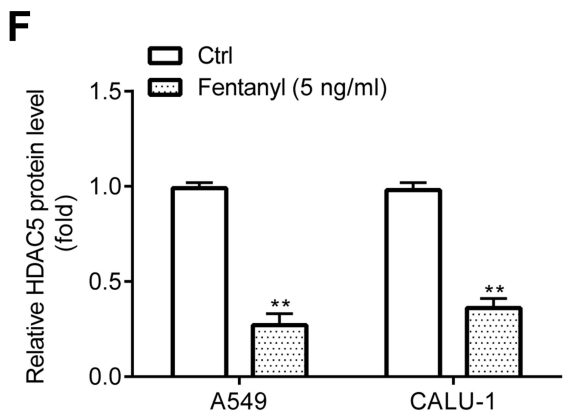

Figure 5 Fentanyl decreases HDAC5 expression in NSCLC cells. RT-qPCR and Western blot were used to detect mRNA and protein levels of HDAC5 in A549 and CALU-I cells $(\mathbf{A}-\mathbf{F}) .{ }^{* *} \mathrm{p}<0.0 \mathrm{I}, \mathrm{A} 549$ or CALU-I cells compared with BEAS-2B; fentanyl compared with ctrl.

lower HDAC5 level relative to siRNA-NC group (Figure 6A-C). siRNA-HDAC5-1 showed relative more inhibitory effects, and was used for the subsequent experiments.

CCK-8 assay exerted that in A549 and CALU-1 cells, miR-331-3p inhibitor reverses the inhibitory effects of fentanyl on viability, which was partially abolished by siRNA-HDAC5 (Figure 6D).

Transwell assay exhibited that in A549 and CALU-1 cells, miR-331-3p inhibitor reverses inhibitory effects of fentanyl on invasion, which was partially abolished by siRNA-HDAC5 (Figure 6E and F).

\section{Decreased miR-33I-3p and Increased HDAC5 Was Found in NSCLC Tumor}

\section{Tissues}

RT-qPCR exerted that miR-331-3p level was lower (Figure 7A), while HDAC5 mRNA level was higher (Figure 7B) in tumor tissues relative to adjacent normal tissues from patients with NSCLC. And there was a negative correlation between miR-331-3p and HDAC5 in patients with NSCLC (Figure 7C).

In addition, as shown in Table 1, mean expression level of miR-331-3p was used as the cut-off to analyze the association between miR-331-3p level (low and high) and clinicopathological characteristics of patients with NSCLC. We observed that, the lower the miR-331-3p level, the higher incidence of lymph node metastasis; in addition, the lower the miR-331-3p level, the higher grade of TNM stage. Altogether, miR-331-3p level was associated with lymph node metastasis $(p=0.022)$ and TNM stage $(\mathrm{p}=0.009)$, however, miR-331-3p level was not associated with gender $(p=0.746)$, age $(p=0.743)$, or histological type $(\mathrm{p}=0.515)$.

\section{Discussion}

It is acknowledged that lung cancer is a highly malignant carcinoma, and the majority of lung cancer-related deaths are resulted from metastasis. ${ }^{5}$ Fentanyl is a widely utilized anesthetic for surgery and cancer therapy which also inhibits cell viability and invasion in numerous cancers, e.g., fentanyl reduces the metastasis in colorectal cancer, ${ }^{10}$ fentanyl inhibits cell growth in gastric cancer by regulation of $\mathrm{NF}-\kappa \mathrm{B},{ }^{11,12}$ fentanyl decreases cell viability and tumor growth in pancreatic cancer cells and cell-transplanted mice. $^{24}$

The function of fentanyl on cancer may be mediated by miRNAs. For example, the inhibitory effects of fentanyl on tumor growth and cell invasion in colorectal cancer may be realized by downregulation of miR-182 through $\beta$-catenin. ${ }^{25}$ In a recent miRNA array in primary cultures of rat hippocampal neurons, fentanyl was proved to increase miR-331-3p level, ${ }^{20}$ which functions as 


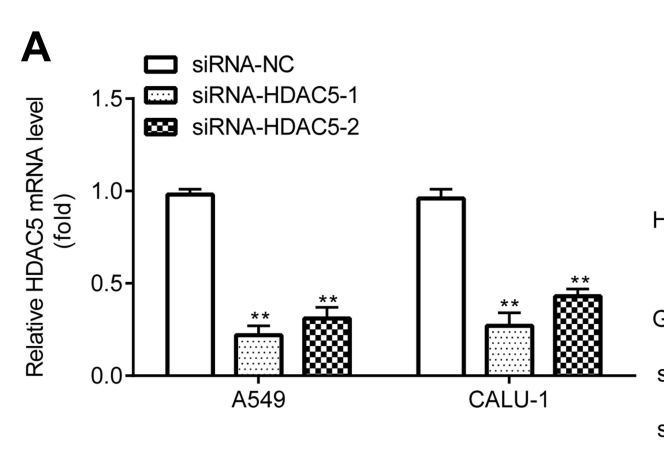

B A549 CALU-1
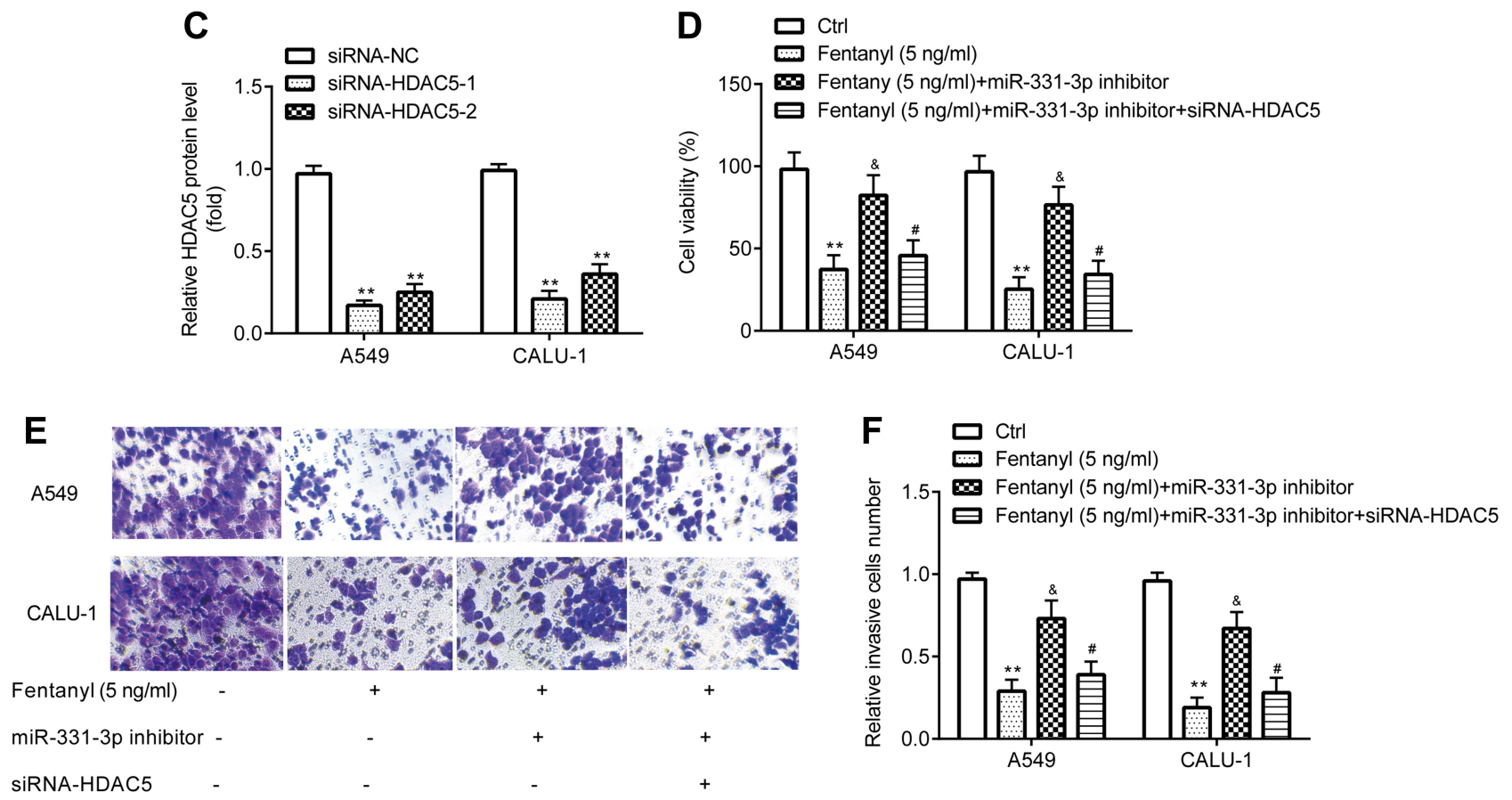

Figure 6 Fentanyl inhibits NSCLC cell viability and invasion by miR-33I-3p/HDAC5. RT-qPCR and Western blot were used to detect mRNA and protein levels of HDAC5 in A549 and CALU-I cells (A-C). A549 and CALU-I cell viability was detected by the CCK-8 assay (D). A549 and CALU-I cell invasion was determined by Transwell assay (E,

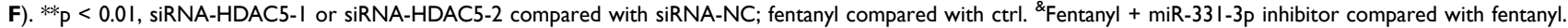
\#Fentanyl + miR-33I-3p inhibitor + siRNA-HDAC5 compared with fentanyl + miR-33I-3p inhibitor.

a tumor suppresser in numerous cancers including lung cancer, e.g., miR-331-3p inhibits proliferation and migration in hepatocellular carcinoma Bel-7402 cell line by targeting E2F1, ${ }^{26}$ miR-331-3p suppresses proliferation and metastasis in ovarian cancer through regulation of $\mathrm{RCC} 2,{ }^{27}$ miR-331-3p reduces cell proliferation in breast cancer through targeting NRP2, ${ }^{28}$ Circular RNA circ_0001649 represses cell proliferation and invasion of NSCLC by sponging miR-331-3p, ${ }^{29}$ miR-331-3p suppresses cell proliferation and invasion of NSCLC by targeting ErbB2 and VAV2. ${ }^{21}$ Furthermore, in 2020, Tian et al reported that miR-331-3p inhibits cell proliferation and invasion of NSCLC cells by targeting MLLT $10 ;{ }^{30}$ circulating miR-331-3p was identified as a novel biomarker for early diagnosis and monitoring of lung cancer. ${ }^{31}$ However, the interaction between fentanyl and miR-331-3p in human disease, including NSCLC has not been revealed yet.

Consistent to previous studies, ${ }^{21,29-31}$ current study observed that miR-331-3p level was lower in NSCLC cell lines than that in BEAS-2B. In addition, miR-331-3p level was increased by fentanyl, and inhibition of miR-331-3p reversed the inhibitory effects of fentanyl on NSCLC cell viability and invasion, indicating the tumor suppressor role of miR-331-3p in NSCLC, as well as the relation between fentanyl and miR-331-3p in the progression of NSCLC.

mRNA targets for miR-331-3p which act as oncogenes during the progression of cancer are left to be investigated. HDAC5 is a well-known oncogene in numerous cancer types including lung cancer, e.g., HDAC5 level is 
A

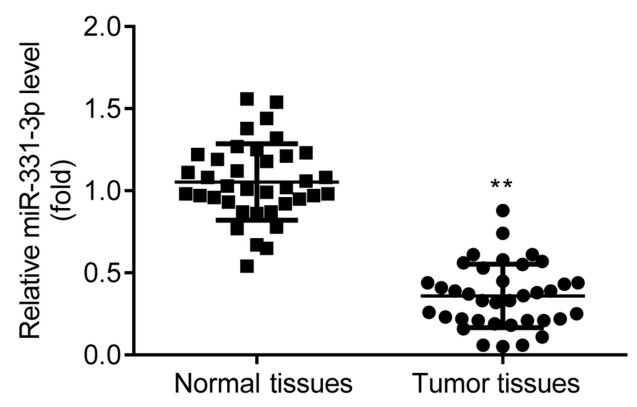

B

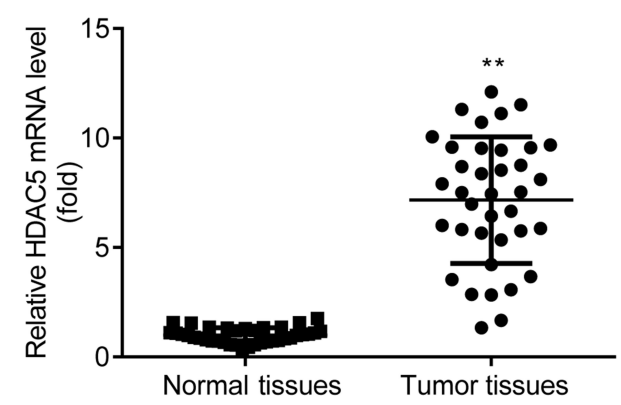

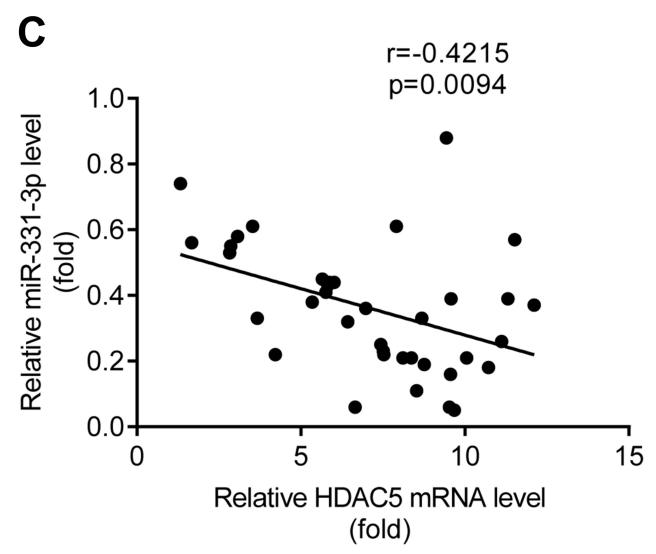

Figure 7 Decreased miR-33I-3p and increased HDAC5 in NSCLC tumor tissues. RT-qPCR was used to detect the expression of miR-33I-3p (A) and HDAC5 mRNA (B) in tumor tissues. Pearson correlation analysis was used to analyze the correlation between miR-33I-3p and HDAC5 in patients with NSCLC (C). ** $p<0.0$ I, tumor tissues compared with healthy tissues.

increased in LCSCs, ${ }^{32}$ HDAC5 induces the malignancy in NSCLC, ${ }^{33}$ HDAC5 increases the cell proliferation and invasion in lung cancer, ${ }^{34}$ HDAC5 is a predictor for poor

Table I Association Between miR-33I-3p Level and Clinicopathological Characteristics of Patients with NSCLC

\begin{tabular}{|c|c|c|c|c|}
\hline Characteristics & Cases & Low & High & $\mathbf{P}$ \\
\hline Gender & & & & 0.746 \\
\hline Male & 20 & 9 & 11 & \\
\hline Female & 17 & 9 & 8 & \\
\hline Age (years) & & & & 0.743 \\
\hline$\leq 55$ & 15 & 8 & 7 & \\
\hline$>55$ & 22 & 10 & 12 & \\
\hline Histological type & & & & 0.515 \\
\hline Squamous cell carcinoma & 20 & 11 & 9 & \\
\hline Adenocarcinoma & 17 & 7 & 10 & \\
\hline Lymph node metastasis & & & & 0.022 \\
\hline Yes & 19 & 13 & 6 & \\
\hline No & 18 & 5 & 13 & \\
\hline TNM stage & & & & 0.009 \\
\hline I-II & 19 & 5 & 14 & \\
\hline III-IV & 18 & 13 & 5 & \\
\hline
\end{tabular}

clinical outcomes in pancreatic neuroendocrine tumor, ${ }^{35}$ HDAC5 inhibitors act as potential therapies for young women with breast cancer. ${ }^{36}$ However, the relation between miR-331-3p and HDAC5 has not been investigated until the conduction of the present study. In the present study, HDAC5 expression was higher in NSCLC cell lines than in BEAS-2B, which was reversed by fentanyl, the findings first indicated the potential relation between fentanyl and HDAC5 in the progression of NSCLC. Besides, HDAC5 was proved to be targeted by miR-331-3p in NSCLC cell lines, miR-331-3p inhibitor reverses inhibitory effects of fentanyl on NSCLC cell viability and invasion, which was partially abolished by siRNA-HDAC5.

At last, miR-331-3p level was lower and HDAC5 mRNA was higher in tumor tissues compared with adjacent normal tissues from the patients with NSCLC, and there was a negative correlation between miR-331-3p and HDAC5 in patients with NSCLC. miR-331-3p level was associated with lymph node metastasis and TNM stage. These findings further indicated the inhibitory role of miR-331-3p and the promotional role of HDAC5 during the progression of NSCLC. 
Taken together, the present study suggested that fentanyl inhibits the viability and invasion of NSCLC cells by increasing miR-331-3p and decreasing HDAC5, which first identifies the relation between fentanyl and miR331-3p, fentanyl and HDAC5 as well as miR-331-3p and HDAC5 in NSCLC. Moreover, during the process of our submission, Luo et al reported the relation between miR331-3p and HDAC5 in vascular diseases in Nov, 2020, which demonstrated that CircSFMBT2 promoted the cell proliferation of vascular smooth muscle cells through targeting miR-331-3p/HDAC5, ${ }^{37}$ which further affirmed the findings in current study.

Whereas, there are 2 limitations in current study: 1) As acknowledged, the overall survival of patients with lung cancer was correlated with the level of natural killer cells (NK cells) cells, ${ }^{38}$ whose reduction generates the initiation and progression of tumors. ${ }^{39}$ Opioid growth factor, one of the endogenous opioids, improves the immune function via elevating NK cells in cancer patients. ${ }^{40}$ In addition, for patients with NSCLC underwent thoracotomy, the percentage and function of NK cells were reduced, which were higher in general preoperative fentanyl anesthesia group than in 24-h postoperative epidural fentanyl analgesia group, indicating the improvement of fentanyl on NK cells. ${ }^{41}$ By survey of literatures, we noticed a report indicating that it is urgent to develop novel NK cell-based therapies for patients with lung cancer. ${ }^{42}$ Consequently, the effect of fentanyl on NK cells in patients with NSCLC will be investigated in our future work; 2) The present study lack the content of animal experiments, which will also be further investigated in our future work.

\section{Disclosure}

The authors declare that they have no competing interests.

\section{References}

1. Torre LA, Siegel RL, Jemal A. Lung cancer statistics. Adv Exp Med Biol. 2016;893:1-19.

2. Zhang HJ, Wang HY, Zhang HT, et al. Transforming growth factor-b1 promotes lung adenocarcinoma invasion and metastasis by epithelial-to-mesenchymal transition. Mol Cell Biochem. 2011;355(1-2):309-314. doi:10.1007/s11010-011-0869-3

3. Meza R, Meernik C, Jeon J, Cote ML. Lung cancer incidence trends by gender, race and histology in the United States, 1973-2010. PLoS One. 2015;10(3):e0121323. doi:10.1371/journal.pone.0121323

4. Haroun RA-H, Zakhary NI, Mohamed MR, Abdelrahman AM, Kandil EI, Shalaby KA. Assessment of the prognostic value of methylation status and expression levels of FHIT, GSTP1 and p16 in non-small cell lung cancer in Egyptian patients. Asian Pac $J$ Cancer Prev. 2014;15(10):4281-4287. doi:10.7314/ APJCP.2014.15.10.4281
5. Kamrava M, Bernstein M, Camphausen K, Hodge J, Gulley J. Combining radiation therapy, immunotherapy, and antiangiogenesis agents in cancer treatment: the 3 musketeers or just another quixotic combination? Radiological Society of North America 2009 Scientific Assembly Annual Meeting; McCormick Place, Chicago.

6. Kumpiro S, Sriuranpong V, Areepium N. Impact of the copper transporter protein 1 (CTR1) polymorphism on adverse events among advanced nonsmall cell lung cancer patients treated with a carboplatin/gemcitabine regimen. Asian Pac J Cancer Prev. 2016;17(9):4391-4394.

7. Qin $\mathrm{Z}, \mathrm{Wu} \mathrm{J}, \mathrm{Xu} \mathrm{C}$, et al. Long-term effects of acupuncture for chronic prostatitis/chronic pelvic pain syndrome: systematic review and single-arm meta-analyses. Ann Transl Med. 2019;7(6):113. doi:10.21037/atm.2018.06.44

8. Gika M, Eguchi K, Inoue Y, Izawa N, Takeuchi K, Nakayama M. Successful management of a patient with pain and dyspnea from bone metastasis and lymphangitis carcinomatosa after surgery for non-small cell lung cancer (NSCLC) in whom optimal dosages of transdermal fentanyl were determined by titration with fentanyl injection, and oxycodone hydrochloride. Gan To Kagaku Ryoho. 2010;37 (3):547-550.

9. Shibahara H, Ando A, Suzuki S, Uematsu N, Nishimura D. Oxycodone and pregabalin using transdermal fentanyl patch provided relief of symptoms for postherpetic neuropathic pain in a patient with non-small cell lung cancer. Gan to Kagaku. 2011;38(10):1675-1677.

10. Li AX, Xin WQ, Ma CG. Fentanyl inhibits the invasion and migration of colorectal cancer cells via inhibiting the negative regulation of Ets-1 on BANCR. Biochem Biophys Res Commun. 2015;465 (3):594-600. doi:10.1016/j.bbrc.2015.08.068

11. Qin Y, Li L, Chen J, et al. Fentanyl inhibits progression of human gastric cancer MGC-803 cells by NF-kappaB downregulation and PTEN upregulation in vitro. Oncol Res. 2012;20(2-3):61-69. doi:10.3727/096504012X13473664562501

12. He G, Li LI, Guan E, Chen J, Qin YI, Xie Y. Fentanyl inhibits the progression of human gastric carcinoma MGC- 803 cells by modulating NF-אB-dependent gene expression in vivo. Oncol Lett. 2016;12 (1):563-571. doi:10.3892/ol.2016.4619

13. Lee HJ. Exceptional stories of microRNAs. Exp Biol Med (Maywood). 2013;238(4):339-343. doi:10.1258/ebm.2012.012251

14. Pratap P, Raza ST, Abbas S, Mahdi F. MicroRNA-associated carcinogenesis in lung carcinoma. $J$ Cancer Res Ther. 2018;14 (2):249-254.

15. Zhu Q, Zang Q, Jiang ZM. Enhanced expression of noncoding miR92a expression is implicated in the development of lung cancer. Eur Rev Med Pharmacol Sci. 2018;22(4):1028-1034.

16. Wiggins JF, Ruffino L, Kelnar K, et al. Development of a lung cancer therapeutic based on the tumor suppressor microRNA-34. Cancer Res. 2010;70(14):5923-5930. doi:10.1158/0008-5472.CAN-10-0655

17. Zhao ZH, Hao W, Meng QT, Du XB, Lei SQ, Xia ZY. Long non-coding RNA MALAT1 functions as a mediator in cardioprotective effects of fentanyl in myocardial ischemia-reperfusion injury. Cell Biol Int. 2017;41(1):62-70. doi:10.1002/cbin.10701

18. Wu Q, Hwang CK, Zheng H, et al. MicroRNA 339 down-regulates $\mu$ opioid receptor at the post-transcriptional level in response to opioid treatment. FASEB J. 2013;27(2):522-535. doi:10.1096/fj.12-213439

19. Zheng H, Chu J, Zeng Y, Loh HH, Law PY, Yang Y. 1 phosphorylation contributes to the differential effects of mu-opioid receptor agonists on microRNA-190 expression. J Biol Chem. 2010;285 (29):21994-22002. doi:10.1074/jbc.M110.112607

20. Zheng H, Zeng Y, Zhang X, Chu J, Loh HH, Law PY. mu-opioid receptor agonists differentially regulate the expression of miR-190 and NeuroD. Mol Pharmacol. 2010;77(1):102-109.

21. Li X, Zhu J, Liu Y, Duan C, Chang R, Zhang C. MicroRNA-331-3p inhibits epithelial-mesenchymal transition by targeting ErbB2 and VAV2 through the Rac1/PAK1/ $\beta$-catenin axis in non-small-cell lung cancer. Cancer Sci. 2019;110:1883-1896. doi:10.1111/cas.14014 
22. Ziesenitz VC, König SK, Mahlke NS, Skopp G, Haefeli WE, Mikus G. Pharmacokinetic interaction of intravenous fentanyl with ketoconazole. J Clin Pharmacol. 2015;55(6):708-717. doi:10.1002/ jcph.469

23. Livak KJ, Schmittgen TD. Analysis of relative gene expression data using real-time quantitative PCR and the 2(-delta delta $\mathrm{C}(\mathrm{T})$ ) method. Methods. 2001;25(4):402-408.

24. Miao J, Wang L, Chen L, Yang T, Jin L, Lin L. Fentanyl inhibits cell viability in human pancreatic cancer cell line and tumor growth in pancreatic cancer cell-transplanted mice. Int J Clin Exp Med. 2015;8 (10):17684-17693.

25. Zhang XL, Chen ML, Zhou SL. Fentanyl inhibits proliferation and invasion of colorectal cancer via $\beta$-catenin. Int J Clin Exp Pathol. 2015;8(1):227-235.

26. Jin W, Zhong N, Wang L, Yu J, Yin F, Zhang K. MiR-331-3p inhibition of the hepatocellular carcinoma (HCC) Bel-7402 cell line by down-regulation of E2F1. J Nanosci Nanotechnol. 2019;19 (9):5476-5482. doi:10.1166/jnn.2019.16535

27. Buranjiang G, Kuerban R, Abuduwanke A, Li X, Kuerban G. MicroRNA-331-3p inhibits proliferation and metastasis of ovarian cancer by targeting RCC2. Arch Med Sci. 2019;15(6):1520-1529. doi:10.5114/aoms.2018.77858

28. Zhao M, Zhang M, Tao Z, Cao J, Wang L, Hu X. miR-331-3p suppresses cell proliferation in TNBC cells by downregulating NRP2. Technol Cancer Res Treat. 2010;19:1533033820905824.

29. Liu T, Song Z, Gai Y. Circular RNA circ_0001649 acts as a prognostic biomarker and inhibits NSCLC progression via sponging miR-331-3p and miR-338-5p. Biochem Biophys Res Commun. 2018;503(3):1503-1509. doi:10.1016/j.bbrc.2018.07.070

30. Tian Q, Xia J, Zhang X, Gao BQ, Wang W. miR-331-3p inhibits tumor cell proliferation, metastasis, invasion by targeting MLLT10 in non-small cell lung cancer. Cancer Manag Res. 2020;12:5749-5758. doi:10.2147/CMAR.S249686

31. Zhang YH, Jin M, Li J, Kong X. Identifying circulating miRNA biomarkers for early diagnosis and monitoring of lung cancer Biochim Biophys Acta Mol Basis Dis. 2020;1866(10):165847. doi:10.1016/j.bbadis.2020.165847
32. Zhao M, Li L, Zhou J, et al. MiR-2861 behaves as a biomarker of lung cancer stem cells and regulates the HDAC5-ERK system genes. Cell Reprogram. 2018;20(2):99-106. doi:10.1089/cell.2017.0045

33. Liu C, Lv D, Li M, et al. Hypermethylation of miRNA-589 promoter leads to upregulation of HDAC5 which promotes malignancy in non-small cell lung cancer. Int J Oncol. 2017;50(6):2079-2090. doi:10.3892/ijo.2017.3967

34. Zhong L, Sun S, Yao S, Han X, Gu M, Shi J. Histone deacetylase 5 promotes the proliferation and invasion of lung cancer cells. Oncol Rep. 2018;40(4):2224-2232.

35. Klieser E, Urbas R, Stättner S, et al. Comprehensive immunohistochemical analysis of histone deacetylases in pancreatic neuroendocrine tumors: HDAC5 as a predictor of poor clinical outcome. Hum Pathol. 2017;65:41-52. doi:10.1016/j.humpath.2017.02.009

36. Oltra SS, Cejalvo JM, Tormo E, et al. HDAC5 inhibitors as a potential treatment in breast cancer affecting very young women. Cancers (Basel). 2020;12(2):E412. doi:10.3390/cancers12020412

37. Luo Y, Huang C. CircSFMBT2 facilitates vascular smooth muscle cell proliferation by targeting miR-331-3p/HDAC5. Life Sci. 2020;118691. doi:10.1016/j.lfs.2020.118691

38. Jin S, Deng Y, Hao JW, et al. NK cell phenotypic modulation in lung cancer environment. PLoS One. 2014;9(10):e109976. doi:10.1371/ journal.pone.0109976

39. Vivier E, Raulet DH, Moretta A, et al. Innate or adaptive immunity? The example of natural killer cells. Science. 2011;331(6013):44-49. doi: $10.1126 /$ science. 1198687

40. Plotnikoff NP, Miller GC, Nimeh N, Faith RE, Murgo AJ, Wybran J. Enkephalins and T-cell enhancement in normal volunteers and cancer patients. Ann N Y Acad Sci. 1987;496(1 Neuroimmune I):608-619. doi:10.1111/j.1749-6632.1987.tb35821.x

41. Cata JP, Bauer M, Sokari T, et al. Effects of surgery, general anesthesia, and perioperative epidural analgesia on the immune function of patients with non-small cell lung cancer. J Clin Anesth. 2013;25 (4):255-262. doi:10.1016/j.jclinane.2012.12.007

42. Aktaş ON, Öztürk AB, Erman B, Erus S, Tanju S, Dilege S. Role of natural killer cells in lung cancer. J Cancer Res Clin Oncol. 2018;144 (6):997-1003. doi:10.1007/s00432-018-2635-3
OncoTargets and Therapy

\section{Publish your work in this journal}

OncoTargets and Therapy is an international, peer-reviewed, open access journal focusing on the pathological basis of all cancers, potential targets for therapy and treatment protocols employed to improve the management of cancer patients. The journal also focuses on the impact of management programs and new therapeutic

Submit your manuscript here: https://www.dovepress.com/oncotargets-and-therapy-journal agents and protocols on patient perspectives such as quality of life, adherence and satisfaction. The manuscript management system is completely online and includes a very quick and fair peer-review system, which is all easy to use. Visit http://www.dovepress.com/ testimonials.php to read real quotes from published authors. 